\title{
Associations with being physically active and the achievement of WHO recommendations on physical activity in people with spinal cord injury
}

\author{
A Rauch ${ }^{1}$, T Hinrichs ${ }^{2}$ and A Cieza ${ }^{1,3,4}$ for the SwiSCI study group ${ }^{5}$
}

\begin{abstract}
Study Design: Secondary data analysis from the cross-sectional survey of the Swiss Spinal Cord Injury Cohort Study.
Objectives: To explore associations with physical activity (PA) levels in people with spinal cord injury (SCI) with the specific aim to identify aspects that potentially explain being physically active (PHYS-ACT) and the achievement of the World Health Organization recommendations on PA.
\end{abstract}

Setting: Community sample $(n=485)$.

Methods: Participants who completely answered four items of the Physical Activity Scale for Individuals with Physical Disabilities were included. Two outcome measures were defined: (1) being PHYS-ACT vs being completely inactive and (2) achieving WHO recommendations on PA (ACH-WHO-REC) (at least $2.5 \mathrm{~h}$ per week of at least moderate intensity) vs performing less. Independent variables were selected from the original questionnaire by applying the ICF framework. Multivariate logistic regression analyses were conducted.

Results: In the participants (aged $52.8 \pm 14.8 ; 73.6 \%$ male) older age decreased, but being a manual wheelchair user increased the odds of achieving both outcomes. Social support and self-efficacy increased the odds of being PHYS-ACT. Use of an intermittent catheter increased, whereas dependency in self-care mobility and coping with emotions decreased the odds for ACH-WHO-REC. Experiencing hindrances due to accessibility is associated with increased odds for ACH-WHO-REC.

Conclusion: Being PHYS-ACT at all and achieving the WHO recommendations on PA are associated with different aspects. Applying the ICF framework contributes to a comprehensive understanding of PA behavior in people with $\mathrm{SCl}$, which can tailor the development of interventions. Longitudinal studies should be initiated to test these associations for causal relationships.

Spinal Cord (2017) 55, 235-243; doi:10.1038/sc.2016.126; published online 16 August 2016

\section{INTRODUCTION}

Physical inactivity is among the most frequent risk factors for mortality in the general population. ${ }^{1}$ For people with spinal cord injury (SCI) living in high-income countries, the leading causes for death shift to those in the general population. ${ }^{2}$ General and SCIspecific guidelines for physical activity (PA) suggest specific types and amounts of PA to prevent diseases related to physical inactivity. The World Health Organization's (WHO) recommendations on PA suggest performing aerobic exercises of at least moderate intensity for at least $150 \mathrm{~min}(2.5 \mathrm{~h})$ per week. ${ }^{1}$ In contrast, the most recent SCI-specific guidelines suggest a minimum of moderate-intensity aerobic exercises for least $20 \mathrm{~min}$ (two times) per week. ${ }^{3}$ However, recent research has shown that this amount might not be sufficient to reduce risks, in particular, for cardiovascular diseases. ${ }^{4}$

Recent research in people living with SCI in Switzerland showed that $81 \%$ are physically active (PHYS-ACT) by performing any leisuretime PA, whereas only $49 \%$ achieve the WHO recommendations for PA (ACH-WHO-REC). ${ }^{5}$ When exploring sociodemographic and SCIrelated characteristics, it was found that people aged 71 years and older and people with complete tetraplegia had significantly lower odds for being PHYS-ACT and to ACH-WHO-REC compared with their respective reference population. Furthermore, the odds to $\mathrm{ACH}$ WHO-REC was lower for women and the odds to be PHYS-ACT was lower when the time since injury was shorter than 5 years. Manual wheelchair users had significantly higher odds to be PHYS-ACT and to ACH-WHO-REC compared with pedestrians without or with devices and users of an electric wheelchair. These findings ask to investigate associations with being PHYS-ACT and the ACH-WHO-REC.

To date, many aspects covering different areas have been found to explain PA levels in people with SCI; ${ }^{6}$ however, comprehensive models have been rarely applied. Given a potential multidimensionality, a comprehensive model that controls for confounding effects among the different domains is useful. The International Classification of Functioning, Disability and Health (ICF) ${ }^{7}$ provides a comprehensive model that is composed of body functions, body structures, activities and participation (referring to functioning) and environmental and personal factors (referring to contextual factors). The ICF Core Sets

\footnotetext{
${ }^{1}$ Department of Medical Informatics, Biometry and Epidemiology_IBE, Chair for Public Health and Health Services Research, Research Unit for Biopsychosocial Health, LudwigMaximilians-University Munich, Munich, Germany; ${ }^{2}$ Division of Sports and Exercise Medicine, Department of Sport, Exercise and Health, University of Basel, Basel, Switzerland; ${ }^{3}$ Swiss Paraplegic Research, Nottwil, Switzerland and ${ }^{4}$ Faculty of Social and Human Sciences, School of Psychology, University of Southampton, Southampton, UK

${ }^{5}$ The members of the SwiSCl Steering Committee are listed before references.

Correspondence: A Rauch, Department of Medical Informatics, Biometry and Epidemiology—IBE, Chair for Public Health and Health Services Research, Research Unit for Biopsychosocial Health, Ludwig-Maximilians-University Munich, Marchioninistrasse 17, Munich 81377, Germany.

E-mail: rauchalex@hotmail.com

Received 21 March 2016; revised 12 July 2016; accepted 14 July 2016; published online 16 August 2016
} 
for $\mathrm{SCI}^{8,9}$ present lists of categories of these components that are relevant to functioning in people with SCI. The ICF framework was first applied in a large quantitative study investigating predictors to explain PA levels in a Canadian population. ${ }^{10}$ For Switzerland, a qualitative study used the ICF and identified associations with participation in PA from all ICF components. ${ }^{11}$ However, these findings have not been validated with quantitative research yet.

Given that various factors from various domains have been found to associate with PA, the strength of the associations with PA may vary and some aspects may influence each other. A comprehensive approach applying multivariate analyses will help to identify those factors from different domains that finally associate with PA and the achievement of WHO recommendations without confounding each other. Thus, the overall objective of this study is to explore associations with PA levels by applying a comprehensive model based on the ICF framework. The specific aim is to identify those aspects that potentially explain being physically active and the achievement of the WHO recommendations on PA.

\section{MATERIALS AND METHODS}

\section{Study design}

This study analyzed data from the Swiss Spinal Cord Injury Cohort Study (SwiSCI) Community Survey 2012, which contained three modules: (1) questionnaire on basic sociodemographics, lesion characteristics, care situation; (2) detailed information on functioning, health, environmental and personal factors; and (3) three different specific modules. ${ }^{12}$ The specific modules covered a 'Psychological Personal Factors and Health Behavior Module' module, a 'Work' module and a 'Health Services Research' module. Participants were recruited via four SCI rehabilitation centers, the national association for people with SCI, an SCI-specific home care institution and a national insurance company. In total, 1549 individuals (traumatic or nontraumatic SCI, 16 years and older) participated in the first two modules of the survey (response rate: $49.3 \%$, median age: 52 years, $71.5 \%$ male, median time since injury: 13.5 years, paraplegia: $69.2 \%$ ). Thereof, 570 individuals were randomly selected to participate in the specific module on 'Psychological Personal Factors and Health Behavior Module', which included questions on PA behavior. Of these, 511 subjects answered this module.

\section{Data collection and item selection}

Sociodemographic and SCI-related characteristics were selected to describe the study population. Furthermore, age, sex, time since onset of SCI, severity of SCI and type of locomotion were included in the regression analysis as they showed associations with either both or one of the two outcomes in a previous study. ${ }^{5}$ The type of locomotion was assessed with item 13 (mobility for moderate distances: $10-100 \mathrm{~m}$ ) of the Self-reported Spinal Cord Independence Measure (SR-SCIM); ${ }^{13}$ the response options for walking with different devices were summarized to 'pedestrian with device or support'.

PA was assessed with four items (items 3-6) of the Physical Activity Scale for Individuals with Physical Disabilities (PASIPD). ${ }^{14}$ These items comprise sport and recreational PAs with light, moderate and strenuous intensity, and muscle strengthening exercises. The total time (hours per week) for each item and for combinations of the different items can be calculated. Moderate- and strenuous-intensity exercises comply with the intensities suggested in the WHO recommendations on PA.

The selection of ICF categories as covariables was based on the Brief ICF Core Set for SCI in the long-term context ${ }^{8}$ as well as on considerations on potential predictors of PA levels. These considerations were informed by results from previous studies on correlates of PA in people with SCI. ${ }^{6,10,11}$ Whenever more than one variable of the questionnaire referred to one selected ICF category, correlations between these variables and the ACH-WHO-REC were calculated. Only the variable with the highest correlation coefficient was selected. Alternatively, a parent item was created for some variables to combine similar information.
Body structures. Body structures were covered with the severity of SCI. No additional body structure was included.

Body functions. Emotional functions were assessed with the SF-36 five-item Mental Health Index ${ }^{15}$ covering five 5-point questions with a sum score from 0 (worst) to 100 (best mental health). Pain, bowel and bladder functions and spasticity were each assessed with a question asking for problems during the past 3 months (response options: no, little or rare, moderate or occasionally, severe or chronic problem).

Activities and participation. Intermittent catheterization was assessed with item $6 \mathrm{~B}$ and self-care mobility with the combined items $2 \mathrm{~A}$ (washing upper body), $2 \mathrm{~B}$ (washing lower body), 3A (dressing upper body), 3B (dressing lower body) and 11 (transferring from wheelchair to toilet/tub) of the SR-SCIM. ${ }^{13}$ Problems in outdoor mobility were assessed with one question on the ability to perform the task (response options: not applicable, not possible, with assistance, with support, without support). Employment was assessed with one question on the amount of hours spent per week (response options: 0, 1-8, 9-16, 17-24, 25-35, 36 or more). Social activities were assessed with one question on the frequency of performing day trips or outdoor activities during the past 4 weeks (response options: $0,1-2,3-5,6-10,11-18,19$ or more).

Environmental factors. Accessibility of public places was measured with item 1, and social attitudes with item 3, of the Nottwil Environmental Factors Inventory Short Form ${ }^{16}$ asking to evaluate the influence of the respective aspect (response options: no influence, made my life a little harder, made my life a lot harder). Social support was assessed with the Social Support Questionnaire. ${ }^{17}$ The Social Support Questionnaire asks participants to name the number of supporters and to score the satisfaction with their support. A sum score is calculated (0 presents no and 90 the highest support).

Personal factors. Self-efficacy was assessed with the General Self-Efficacy Scale, ${ }^{18}$ which includes ten 4 -point items with a total score from 10 to 40 (higher scores meaning better self-efficacy). The Purpose in Life Test-Short Form (PIL-SF $)^{19}$ was included to address general life goals as an indicator for goal orientation. The PIL-SF comprises four 7-point items with a total score from 4 to 28 (higher scores suggest greater purpose in life). To address coping, the sub-scale coping with emotions from the Brief $\mathrm{Cope}^{20}$ was included as it showed the highest correlation with ACH-WHO-REC among all scales. This scale summarizes two questions referring to focusing and venting of emotions. The score ranges from 2 to 8 (higher scores meaning increased tendency to apply the strategy).

\section{Data analyses}

Descriptive statistics were conducted to describe the study population and to calculate the proportion of those who were PHYS-ACT and those who achieved the WHO recommendations on PA (at least $2.5 \mathrm{~h}$ of moderate and strenuous PAs per week).

Multivariate logistic regression analyses were performed with PHYS-ACT and the ACH-WHO-REC as dependent variables. To prepare data for regression analyses, response options of independent variables with ordinal scales were dichotomized into groups of people who had the corresponding outcome versus those who did not. Hierarchical models entering blocks successively were calculated for the two dependent variables. The first block (sociodemographic and SCI-related variables) consisted of variables that were identified to be independently associated with the respective PA outcome in the previous study. ${ }^{5}$ Then, blocks of independent variables referring to each ICF component (body functions, activities and participation, environmental factors, personal factors) were added in a stepwise manner. Thus, the final model included all independent variables.

Missing values in the independent variables were addressed by conducting multiple imputations. Therefore, all variables included in the regression models were entered, and five iterations were performed. In all regression models, odds ratios with $95 \%$ confidence intervals and the corresponding $P$-value were calculated. The level of significance was set as $P<0.05$. The $R^{2}$ (Nagelkerke) was calculated for goodness-of-fit analyses. Statistical analyses were performed with SPSS 21 (SPSS Inc., Chicago, IL, USA). 
The survey has been performed in accordance with the ethical standards according to the Declaration of Helsinki and has been approved by the Ethics Committee of the Canton of Lucerne. All participants gave written informed consent.

\section{RESULTS}

In total, 485 participants answered the PASIPD completely and were included in this study (Table 1). No differences in sociodemographic and SCI-related characteristics between the respondents and

Table 1 Characteristics of the study population $(n=485)$ and proportions of people being PHYS-ACT and achieving WHO recommendations on physical activity ( $\geqslant 2.5 \mathrm{~h}$ per week of at least moderate intensity)

\begin{tabular}{|c|c|c|c|}
\hline & n (\%) & PHYS-ACT, n (valid \%) & ACH-WHO-REC, n (valid \%) \\
\hline & & & \\
\hline Total sample & $485(100 \%)$ & $395(81.4)$ & 237 (48.9) \\
\hline \multicolumn{4}{|l|}{ Sex } \\
\hline Male & 357 (73.6) & $292(81.8)$ & $184(51.5)$ \\
\hline Female & $128(26.4)$ & $103(80.5)$ & $53(41.4)^{a}$ \\
\hline Missing & $0(0.0)$ & & \\
\hline \multicolumn{4}{|l|}{ Age (years) } \\
\hline & & & \\
\hline & & & \\
\hline $17-30$ & $41(8.5)$ & $39(95.1)$ & $25(61.0)$ \\
\hline $31-50$ & $170(35.1)$ & $139(81.8)$ & $88(51.8)$ \\
\hline $51-70$ & $219(45.2)$ & $178(81.3)$ & $106(48.4)$ \\
\hline$\geqslant 71$ & $55(11.3)$ & $39(70.9)^{a}$ & $18(32.7)^{\mathrm{a}}$ \\
\hline Missing & $0(0.0)$ & & \\
\hline \multicolumn{4}{|l|}{ Years of education (years) } \\
\hline & & & \\
\hline & & & \\
\hline$<13$ & $167(34.4)$ & $127(76.0)$ & $72(43.1)$ \\
\hline 13 & $307(63.3)$ & $258(84.0)$ & $160(52.1)$ \\
\hline Missing & $11(2.3)$ & & \\
\hline \multicolumn{4}{|l|}{ Etiology of SCl } \\
\hline Traumatic & $380(78.7)$ & $313(82.4)$ & $193(50.8)$ \\
\hline Non-traumatic & $103(21.3)$ & $80(77.7)$ & $44(42.7)$ \\
\hline Missing & $2(0.4)$ & & \\
\hline \multicolumn{4}{|l|}{ Time since injury (in years) } \\
\hline & & & \\
\hline & & & \\
\hline $0-5$ & $99(20.4)$ & $75(75.8)$ & $46(46.5)$ \\
\hline $6-15$ & $155(32.0)$ & $134(86.5)^{\mathrm{a}}$ & $78(50.3)$ \\
\hline $16-25$ & $108(22.3)$ & $86(79.6)$ & $60(55.6)$ \\
\hline$\geqslant 26$ & $118(24.3)$ & $96(81.4)$ & $52(44.1)$ \\
\hline Missing & $5(1.0)$ & & \\
\hline \multicolumn{4}{|l|}{ Severity of $\mathrm{SCl}$} \\
\hline Incomplete paraplegia & $169(35.0)$ & $140(82.8)$ & $92(57.9)$ \\
\hline Complete paraplegia & 159 (32.9) & $137(86.2)$ & $79(46.7)$ \\
\hline Incomplete tetraplegia & $100(20.7)$ & $76(76.0)$ & $20(36.4)^{a}$ \\
\hline Complete tetraplegia & $55(11.4)$ & $40(72.7)^{a}$ & $45(45.0)$ \\
\hline Missing & $2(0.4)$ & & \\
\hline \multicolumn{4}{|c|}{ Type of locomotion (when moving around for 10-100 m) } \\
\hline Pedestrian (neither device nor assistance) & $70(15.0)$ & $54(77.1)$ & $27(38.6)$ \\
\hline Pedestrian (with device or assistance) & $91(19.4)$ & $67(73.6)$ & $37(40.7)$ \\
\hline Manual wheelchair (no assistance) & $218(46.6)$ & $199(91.3)^{\mathrm{a}}$ & $134(61.5)^{\mathrm{a}}$ \\
\hline Electric wheelchair/manual with assistance & $89(19.0)$ & $61(68.5)$ & $31(34.8)$ \\
\hline Missing & $17(3.5)$ & & \\
\hline
\end{tabular}

Abbreviations: ACH-WHO-REC, achievement of WHO recommendations on physical activity; PHYS-ACT, physically active; SCl, spinal cord injury; WHO, World Health Organization.

aFor these subgroups, significant differences in being PHYS-ACT and achieving WHO recommendations on physical activity have been found in a previous study. ${ }^{5}$ 
the excluded subjects and non-respondents of the survey have been found.

Table 2 presents an overview of the selected items and the instruments used in the survey to assess these items. Furthermore, it presents the dichotomization of the ordinal variables into each two answer categories and the assessment results (mean value, respectively number of persons referring to each of the answer categories).

Table 3 presents the results for the logistic regression for being PHYS-ACT. The first model shows the significantly decreased and increased odds for being PHYS-ACT for the already known sociodemographic and SCI-related characteristics. While no variable from body functions and activities and participation associated with being PHYS-ACT in the following models, the environmental factors social support and the personal factor higher self-efficacy significantly increased the odds to be PHYS-ACT. From the first model, only the use of a manual wheelchair remained a significant association in the final model; increasing age was still close to the defined significance level. With each stepwise entering of the different blocks, the model fit presented with the Nagelkerke $R^{2}$ increased. The final model explained $25 \%$ of the variance.

Table 4 presents the results for the analyses for ACH-WHO-REC. As in the previous analyses, no body function was found to explain $\mathrm{ACH}-\mathrm{WHO}-\mathrm{REC}$. In contrast, from activities and participation the use of an intermittent catheter significantly increased, and being dependent in self-care mobility significantly decreased the odds to ACH-WHOREC. From environmental factors, experiencing hindrances due to accessibility was significantly associated with ACH-WHO-REC and the personal factors coping with emotions significantly decreased the odds to ACH-WHO-REC. From the first model, only age and the use of a manual wheelchair remained significantly associated with ACH-WHOREC in the final model: older age decreased and the use of a manual wheelchair increased the odds to ACH-WHO-REC. The stepwise

Table 2 Overview of the applied instruments used to assess the independent variables, the dichotomization of ordinal variables and the assessment results $(n=485)$

\begin{tabular}{|c|c|c|c|c|}
\hline Item & $\begin{array}{l}\text { Assessment instruments (for the metric variables the total } \\
\text { score is presented in brackets) }\end{array}$ & Mean (s.d.) & $\begin{array}{l}\text { Dichotomized answer categories } \\
\text { for ordinal variables }\end{array}$ & n (valid \%) \\
\hline \multicolumn{5}{|l|}{$B F$} \\
\hline Emotional functions & Five-item MHI-SF3615 (0-100) & $72.5( \pm 18.0)$ & & \\
\hline \multirow[t]{2}{*}{ Pain } & Question on severity of chronic pain problem & & No pain ${ }^{a}$ & $130(28.2)$ \\
\hline & & & Pain & $331(71.8)$ \\
\hline \multirow[t]{2}{*}{ Bowel and/or bladder problems } & Question on frequency of bladder problems & & No problem ${ }^{a}$ & 90 (19.9) \\
\hline & Question on fecal incontinence & & Bowel and/or bladder problems & $363(80.1)$ \\
\hline \multirow[t]{2}{*}{ Spasticity } & Question on frequency of spasticity & & No spasticitya & $125(27.2)$ \\
\hline & & & Spasticity & $335(72.8)$ \\
\hline \multicolumn{5}{|l|}{$A P$} \\
\hline \multirow[t]{2}{*}{ Use of intermittent catheter } & SR-SCIM 14: Use of intermittent catheter & & No use ${ }^{a}$ & $286(62.2)$ \\
\hline & & & Use & $174(37.8)$ \\
\hline \multirow[t]{5}{*}{ Dependent in self-care mobility } & SR-SCIM 2A: Washing upper body & & Independent ${ }^{a}$ & $141(29.7)$ \\
\hline & SR-SCIM 2B: Washing lower body & & Any dependency in at least one of & $333(70.3)$ \\
\hline & SR-SCIM 3A: Dressing upper body & & the five items & \\
\hline & SR-SCIM 3B: Dressing lower body & & & \\
\hline & SR-SCIM 11: Transfer wheelchair-toilet & & & \\
\hline \multirow[t]{2}{*}{ Difficulties in outdoor mobility } & Question on difficulties in outdoor mobility & & No difficultya & $167(36.0)$ \\
\hline & & & Difficulty & $297(64.0)$ \\
\hline \multirow[t]{2}{*}{ Employment } & Question on the amount of hours of paid work per week & & Unemployed $(0 \mathrm{~h})^{\mathrm{a}}$ & $210(47.2)$ \\
\hline & & & Employed ( >0 h) & $235(52.8)$ \\
\hline \multirow[t]{2}{*}{ Social activities } & Number of social activities outside of the home during the & & 2 times or less ${ }^{a}$ & $201(43.1)$ \\
\hline & past 4 weeks & & $\geqslant 3$ times & $265(56.9)$ \\
\hline \multicolumn{5}{|l|}{$E F$} \\
\hline \multirow[t]{2}{*}{ Hindrance due to accessibility } & Question on the influence of accessibility of public buildings & & No influence ${ }^{a}$ & $169(36.0)$ \\
\hline & & & Hindrance & $300(64.0)$ \\
\hline \multirow[t]{2}{*}{ Hindrance due to social attitudes } & Question on the influence of negative social attitudes of the & & No influence ${ }^{a}$ & $350(75.4)$ \\
\hline & society & & Hindrance & $114(24.6)$ \\
\hline Social support & Social Support Questionnaire ${ }^{17}(0-90)$ & $25.3( \pm 10.4)$ & & $485(100.0)$ \\
\hline \multicolumn{5}{|l|}{$P F$} \\
\hline Self-efficacy & General Self-Efficacy Scale ${ }^{18}(10-40)$ & $30.4( \pm 5.6)$ & & 462 \\
\hline Purpose in life & Purpose in Life-Short Form ${ }^{19}(4-28)$ & $21.3( \pm 4.7)$ & & 472 \\
\hline Coping with emotions & Sum of items 9 and 21 of the Brief Cope ${ }^{20}(2-8)$ & $3.9( \pm 1.4)$ & & 472 \\
\hline
\end{tabular}

Abbreviations: AP, activities and participation; BF, body functions, EF, environmental factors; MHI-SF36, Mental Health Index of the Short Form; ${ }^{15}$ PF, personal factors; s.d., standard deviation; SR-SCIM, Self-Reported Spinal Cord Independence Measure. ${ }^{13}$

aReference categories for the dichotomized variables in the regression models (presented in Tables 3 and 4). 


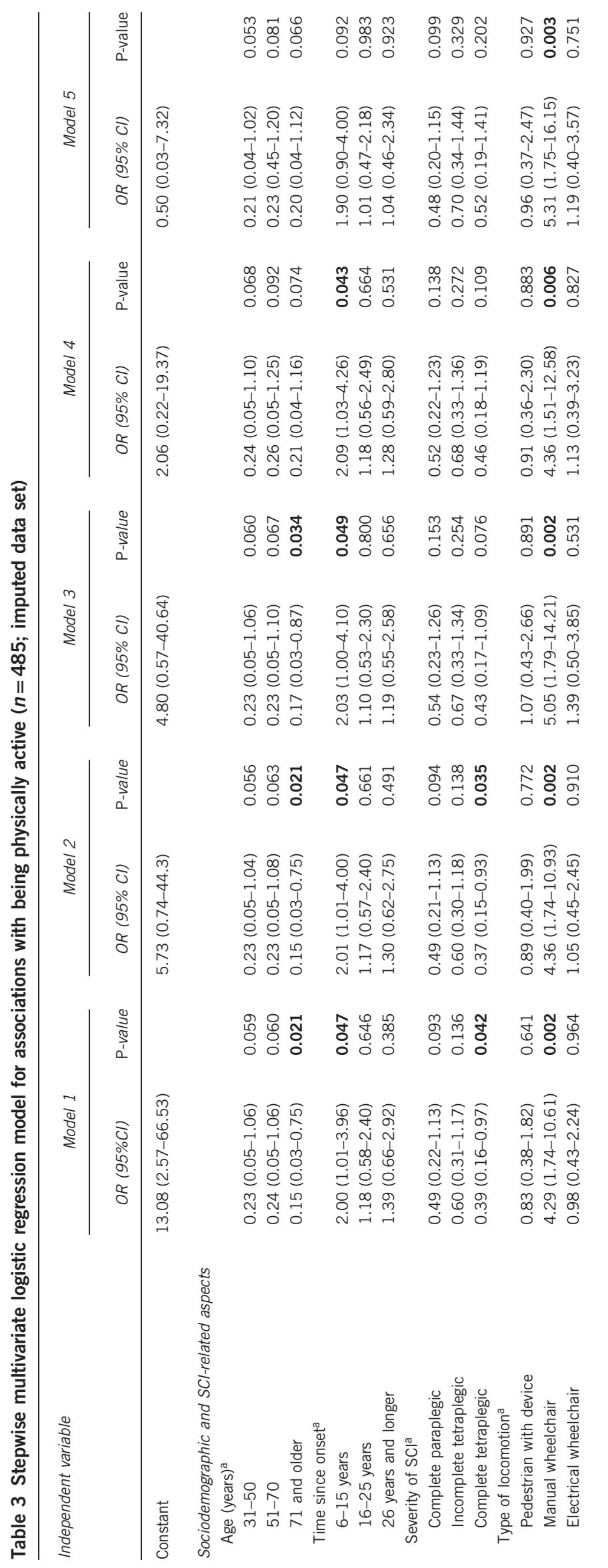

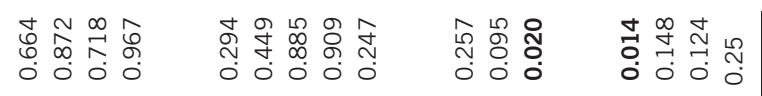

নิ

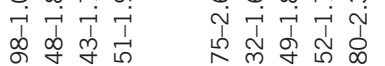

○ㅇำ

ब.

○ं 0 ○ं

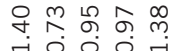

สิ

ำ ㄱํㅇ

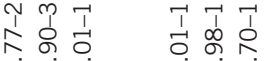

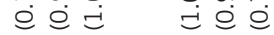

亦

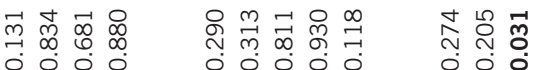

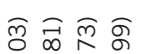

ดำสำ

ธิธ

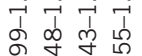

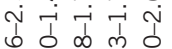

ำ

○อ 0

○े @ᄋ 巳

oㅇ

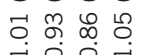

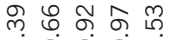

ㅇำ

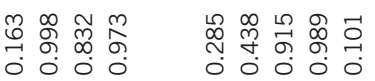

ํㅡ응

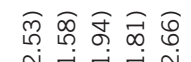

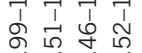

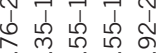

อ 00

다 하

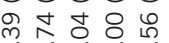

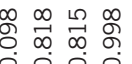

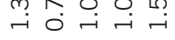

$\circ 000$

$\widehat{\bar{\nu}} \underset{\sim}{\widehat{m}} \underset{\infty}{\widehat{\infty}} \underset{\infty}{0}$

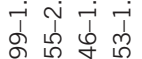

อ อ อ

ㄷํㅇำ

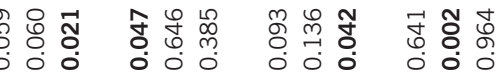

$$
\text { तi }
$$




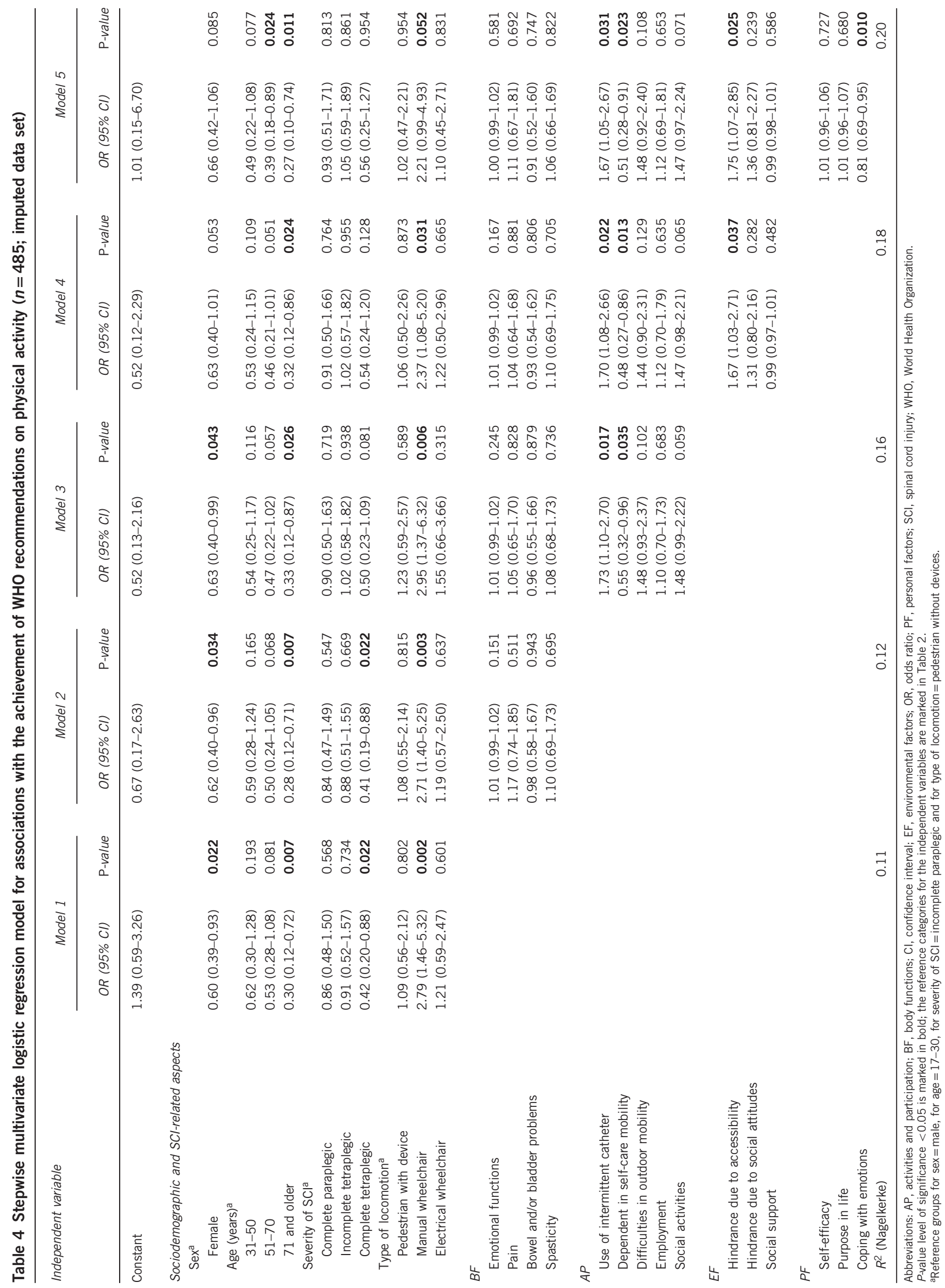


adding of blocks to the first model again increased the model fit with a $R^{2}$ of $20 \%$ in the final model.

\section{DISCUSSION}

Within the use of a comprehensive model based on the ICF framework, we identified a number of aspects from all components, except from body functions, to associate with PHYS-ACT and the ACH-WHO-REC. Only the type of locomotion was significantly associated with both outcomes.

Being a manual wheelchair user increases the odds for both, being PHYS-ACT and for ACH-WHO-REC, compared to moving around as a pedestrian without using devices or support. Generally, it was already found that PA levels in people who are pedestrians are lower compared to those of a manual wheelchair user. ${ }^{21}$ This is relevant for two reasons: First, the finding points out that non-wheelchair users require special attention both in the context of future research as well as for intervention planning. A previous study found that psychosocial factors and the experience of pain may explain the lower levels; however, this is the only evidence yet. ${ }^{5,22}$ Second, most research investigated the severity of SCI to explain PA levels and agrees that tetraplegia is associated with lower PA levels compared with paraplegia. ${ }^{5,21,23-25}$ The association between the type of locomotion and PA levels, however, is rarely investigated. Furthermore, people with SCI not being wheelchair dependent have not been included in all existing studies. Thus, our finding suggests including type of locomotion as a potential predictor for being PHYS-ACT and ACHWHO-REC when investigating PA levels.

In addition to the type of locomotion, older age decreased the odds to being PHYS-ACT ( $P$-values for the three categories nearly reached significance value). Furthermore, only contextual factors explained the outcome. Both higher social support and higher levels of self-efficacy increased the odds of being PHYS-ACT. Social support in people with SCI is related to physical and mental health, pain, coping, adjustment and life satisfaction. ${ }^{26}$ The finding that more social support increases the odds to be PHYS-ACT confirms findings from qualitative studies, ${ }^{11,27-29}$ but differs from the only quantitative study where social support was not found to associate with being PHYS-ACT. ${ }^{10}$ Taking the different findings into account, we assume that whether social support explains being PHYS-ACT in people with SCI depends as well on how people overcome a potential lack of social support. The evaluation of the importance, respectively the strength of intentions to perform physical activities that were found to associate with being PHYS-ACT ${ }^{10,23}$ - may thereby have an important role.

The finding that higher scores in self-efficacy increase the odds of being PHYS-ACT agrees with existing evidence. ${ }^{30}$ General self-efficacy is defined as the 'general beliefs in one's ability to respond to and control environmental demands and challenges'. ${ }^{18}$ In addition to the evidence that better self-efficacy is associated with higher PA levels, self-efficacy is considered an intervention target in health-promoting programmes. ${ }^{31,32}$ Thus, our finding underscores the importance of considering self-efficacy when aiming to empower people with SCI to become or maintain PHYS-ACT.

The ACH-WHO-REC was associated with a larger number of aspects than being PHYS-ACT. The fact that older age decreased the odds for ACH-WHO-REC is well understood as performing increased intensities of physical activities becomes more difficult with age. Increased odds for ACH-WHO-REC were found for using an intermittent catheter. This confirms findings of a previous study that showed that intermittent catheterization is significantly associated with better mobility and social integration compared with those using an indwelling catheter. ${ }^{33}$ The use of indwelling catheters or urinal condoms (in men) requires one to carry a urine bag, which might be inconvenient and prevents people from performing physical activities. ${ }^{34}$ However, people with limited hand functions, for whom the handling of intermittent catheters is rather difficult, are limited in using intermittent catheterization. Application of bladder management that facilitates performing physical activities might present a challenge for them.

Being dependent in self-care mobility decreased the odds for $\mathrm{ACH}-\mathrm{WHO}-\mathrm{REC}$ by about half. Based on a previous qualitative study, ${ }^{11}$ we assume that spending considerable time for physical activities as required to ACH-WHO-REC is limited because of the increased time consumption for self-care when dependent, a lack of wheelchair-accessible toilets in public places, which might increase dependence, a lack of supportive persons, or to not wanting to take advantage of support for more time than necessary within routine selfcare. However, these assumptions are not proven and require additional research.

The finding that the experience of hindrances due to accessibility was positively associated with ACH-WHO-REC suggests that people who are more PHYS-ACT experience more physical barriers. Importantly, this does not prevent them from being PHYS-ACT. In previous qualitative studies, lack of accessibility was reported as a barrier to participating in PA. ${ }^{11,27-29,35-37}$ Thus, different contexts may contribute to different findings, although more quantitative research including comparisons across different countries is required to validate this assumption. In our study, the question on hindrance due to accessibility did not specify distinct types of buildings. Thus, there is no information on the accessibility of sport facilities. To better understand accessibility in the context of PA, specific questions on accessibility of sport facilities $^{38,39}$ should be complemented in the future.

Finally, higher scores in coping with emotions decreased the odds for ACH-WHO-REC. This coping strategy is described as 'the tendency to focus on whatever distress or upset one is experiencing and to ventilate those feelings', whereas this tendency is considered rather maladaptive for successful active coping strategies. ${ }^{40}$ In general, different coping strategies contribute to psychosocial adaptation in people with $\mathrm{SCI}^{41}$ and thus could contribute to participation in PA. In our study, only coping with emotions was included in the analyses because of the given preselection. This personal factor presented the strongest association with ACH-WHO-REC and suggests that people who mentally focus on and ventilate emotional problems are prevented from active coping, and thus from performing physical activities with sufficient duration and intensity. Thus, identifying people with the tendency to focus on their emotions could present a first step before offering strategies to overcome this coping style and replace it by increased physical activities.

None of the investigated body functions was found to associate with one of the two PA outcomes. This confirms the finding for pain and emotional functions from a Canadian study, ${ }^{10}$ but differs from a study in Taiwan where PA was associated with less pain and fewer depressive symptoms. ${ }^{42}$ Importantly, the identification of associations with pain and spasticity can be influenced by the fact that pain and spasticity can both be improved and be aggravated by PA. ${ }^{11}$ To overcome this limitation and to better understand the associations, the quality of the relationships should be assessed in future assessments.

Some limitations related to our study need to be mentioned. Based on the cross-sectional design of the study we cannot draw any conclusions regarding causal relationships. Furthermore, data on PA were collected with a self-report questionnaire, for which it is known that the PA levels are rather overestimated. ${ }^{43}$ Importantly, to date, 
evidence is lacking on whether the ACH-WHO-REC indeed reduces the risk for cardiovascular diseases in people with SCI. Future research is required to confirm this, in order to determine PA levels that are appropriate for people with SCI. Finally, the inclusion of potential aspects that additionally could associate with the ACH-WHO-REC was limited because of the fact that this study presented a secondary data analysis.

The application of the ICF framework contributed to a comprehensive understanding of PA levels in people with SCI. Except body functions, aspects from all components presented associations with the two outcomes. When aiming to empower people to become or remain PHYS-ACT at all, social support and self-efficacy are significant aspects to consider. However, when aiming to empower people to achieve the WHO recommendations on PA, other aspects come to the fore. Then, dependence during self-care and coping with emotions should become intervention targets. Independently from the type of PA, people with SCI not using a wheelchair should receive specific attention in both future research and intervention planning for promoting PA. Longitudinal studies should be initiated to test these associations for causal relationships.

\section{DATA ARCHIVING}

There were no data to deposit.

\section{CONFLICT OF INTEREST}

The authors declare no conflict of interest.

\section{ACKNOWLEDGEMENTS}

This study was financed in the framework of the Swiss Spinal Cord Injury Cohort Study (http: //www.swisci.ch), supported by the Swiss Paraplegic Foundation.

\section{MEMBERS OF THE SWISCI STEERING COMMITTEE}

Xavier Jordan, Bertrand Léger (Clinique Romande de Réadaptation, Sion); Michael Baumberger, Hans Peter Gmünder (Swiss Paraplegic Center, Nottwil); Armin Curt, Martin Schubert (University Clinic Balgrist, Zürich); Margret Hund-Georgiadis, Kerstin Hug (REHAB Basel, Basel); Hans Georg Koch, (Swiss Paraplegic Association, Nottwil); Hardy Landolt (Representative of persons with SCI, Glarus); Hansjörg Koch (SUVA, Luzern); Mirjam Brach, Gerold Stucki (Swiss Paraplegic Research, Nottwil); Martin Brinkhof, Christine Thyrian (SwiSCI Study Center at Swiss Paraplegic Research, Nottwil).

1 World Health Organization (WHO). Global Recommendations on Physical Activity for Health. WHO: Geneva, Switzerland, 2010.

2 World Health Organization (WHO). International Perspectives on Spinal Cord Injury. WHO: Geneva, Switzerland, 2013.

3 Martin Ginis KA, Hicks AL, Latimer AE, Warburton DE, Bourne C, Ditor DS et al. The development of evidence-informed physical activity guidelines for adults with spinal cord injury. Spinal Cord 2011; 49: 1088-1096.

4 Totosy de Zepetnek JO, Pelletier CA, Hicks AL, MacDonald MJ. Following the physical activity guidelines for adults with spinal cord injury for 16 weeks does not improve vascular health: a randomized controlled trial. Arch Phys Med Rehabil 2015; 96: 1566-1575.

5 Rauch A, Hinrichs T, Oberhauser C, Cieza A. Do people with spinal cord injury meet the WHO recommendations on physical activity? Int J Public Health 2016; 61: 17-27.

6 Fekete C, Rauch A. Correlates and determinants of physical activity in persons with spinal cord injury: a review using the International Classification of Functioning, Disability and Health as reference framework. Disabil Health $\mathrm{J}$ 2012; 5: 140-150.

7 World Health Organization (WHO). International Classification of Functioning, Disability and Health. WHO: Geneva, Switzerland, 2001.

8 Cieza A, Kirchberger I, Biering-Sorensen F, Baumberger M, Charlifue S, Post MW et al. ICF Core Sets for individuals with spinal cord injury in the long-term context. Spinal Cord 2010; 48: 305-312.
9 Kirchberger I, Cieza A, Biering-Sorensen F, Baumberger M, Charlifue S, Post MW et al. ICF Core Sets for individuals with spinal cord injury in the early post-acute context. Spinal Cord 2010; 48: 297-304.

10 Martin Ginis KA, Arbour-Nicitopoulos KP, Latimer-Cheung AE, Buchholz AC, Bray SR, Craven $\mathrm{BC}$ et al. Predictors of leisure time physical activity among people with spinal cord injury. Ann Behav Med 2012; 44: 104-118.

11 Rauch A, Fekete C, Cieza A, Geyh S, Meyer T. Participation in physical activity in persons with spinal cord injury: a comprehensive perspective and insights into gender differences. Disabil Health J 2013; 6: 165-176.

12 Fekete C, Segerer W, Gemperli A, Brinkhof MW. Participation rates, response bias and response behaviours in the community survey of the Swiss Spinal Cord Injury Cohort Study (SwiSCI). BMC Med Res Methodol 2015; 15: 80.

13 Fekete C, Eriks-Hoogland I, Baumberger M, Catz A, Itzkovich M, Luthi $\mathrm{H}$ et al. Development and validation of a self-report version of the Spinal Cord Independence Measure (SCIM III). Spinal Cord 2013; 51: 40-47.

14 Washburn RA, Zhu W, McAuley E, Frogley M, Figoni SF. The physical activity scale for individuals with physical disabilities: development and evaluation. Arch Phys Med Rehabil 2002; 83: 193-200.

15 Ware JE Jr, Sherbourne CD. The MOS 36-item short-form health survey (SF-36). I. Conceptual framework and item selection. Med Care 1992; 30: 473-483.

16 Ballert CS, Post MW, Brinkhof MW, Reinhardt JD. Psychometric properties of the Nottwil Environmental Factors Inventory Short Form. Arch Phys Med Rehabil 2015; 96 : 233-240.

17 Sarason I, Sarason B, Shearing E, Pierce G. A brief measure of social support: practical and theoretical implications. J Soc Pers Relat 1987; 4: 497-510.

18 Schwarzer R, Jerusalem M. Generalized self-efficacy scale. In: Weinman J, Wright SMJ (eds). Measures in Health Psychology: A User's Portfolio Causal and Control Beliefs. Windsor, UK, 1995, pp 35-37.

19 Schulenberg SE, Schnetzer LW, Buchanan EM. The purpose in life test-short form: development and psychometric support. J Happiness Stud 2011; 12: 861-876.

20 Carver CS. You want to measure coping but your protocol's too long: consider the brief COPE. Int J Behav Med 1997; 4: 92-100.

21 Martin Ginis KA, Latimer AE, Arbour-Nicitopoulos KP, Buchholz AC, Bray SR, Craven $\mathrm{BC}$ et al. Leisure time physical activity in a population-based sample of people with spinal cord injury part I: demographic and injury-related correlates. Arch Phys Med Rehabil 2010; 91: 722-728.

22 Martin Ginis K, Papathomas A, Perrier M, Smith B, Group S-SR. Psychosocial factors associated with physical activity in ambulatory and manual wheelchair users with spinal cord injury: a mixed-methods study. Disabil Rehabil (e-pub ahead of print 29 June 2015; doi:10.3109/09638288.2015.1045991).

23 Rauch A, Fekete C, Oberhauser C, Marti A, Cieza A. Participation in sport in persons with spinal cord injury in Switzerland. Spinal Cord 2014; 52: 706-711.

24 Anneken V, Hanssen-Doose A, Hirschfeld S, Scheuer T, Thietje R. Influence of physical exercise on quality of life in individuals with spinal cord injury. Spinal Cord 2010; 48: 393-399.

25 van den Berg-Emons RJ, Bussmann JB, Haisma JA, Sluis TA, van der Woude LH, Bergen MP et al. A prospective study on physical activity levels after spinal cord injury during inpatient rehabilitation and the year after discharge. Arch Phys Med Rehabil 2008; 89: 2094-2101.

26 Muller R, Peter C, Cieza A, Geyh S. The role of social support and social skills in people with spinal cord injury-a systematic review of the literature. Spinal Cord 2012; 50: 94-106.

27 Wahman K, Biguet G, Levi R. What promotes physical activity after spinal cord injury? An interview study from a patient perspective. Disabil Rehabil 2006; 28 : 481-488.

28 Kehn M, Kroll T. Staying physically active after spinal cord injury: a qualitative exploration of barriers and facilitators to exercise participation. BMC Public Health 2009; 9: 168.

29 Vissers M, van den Berg-Emons R, Sluis T, Bergen M, Stam H, Bussmann H. Barriers to and facilitators of everyday physical activity in persons with a spinal cord injury after discharge from the rehabilitation centre. J Rehabil Med 2008; 40: 461-467.

30 Kroll T, Kratz A, Kehn M, Jensen M, Groah S, Ljungberg I et al. Perceived exercise selfefficacy as a predictor of exercise behavior in individuals aging with spinal cord injury. Am J Phys Med Rehabil 2012; 91: 640-651.

31 Arbour-Nicitopoulos KP, Martin Ginis KA, Latimer AE. Planning, leisure-time physical activity, and coping self-efficacy in persons with spinal cord injury: a randomized controlled trial. Arch Phys Med Rehabil 2009; 90: 2003-2011.

32 Haworth J, Thornton E. The effects of an 'exercise and education' programme on exercise self-efficacy and levels of independent activity in adults with acquired neurological pathologies: an exploratory, randomized study. Clin Rehabil 2009; 23 : 371-383.

33 Cameron AP, Wallner LP, Forchheimer MB, Clemens JQ, Dunn RL, Rodriguez G et al. Medical and psychosocial complications associated with method of bladder management after traumatic spinal cord injury. Arch Phys Med Rehabil 2011; 92: 449-456.

34 Engkasan JP, Ng CJ, Low WY. Factors influencing bladder management in male patients with spinal cord injury: a qualitative study. Spinal Cord 2014; 52: 157-162.

35 Martin K, Latimer A, Francover C, Hanley H, Watson K, Hicks A et al. Sustaining exercise motivation and participation among people with spinal cord injury. Palaestra 2002; 18: 38-51. 
36 Tasiemski T, Kennedy P, Gardner B, Blaikley R. Athletic identity and sports participation in people with spinal cord injury. Adapt Phys Activ Q 2004; 21: 364-378.

37 Levins SM, Redenbach DM, Dyck I. Individual and societal influences on participation in physical activity following spinal cord injury: a qualitative study. Phys Ther 2004; 84: 496-509.

38 Arbour-Nicitopoulos KP, Martin Ginis KA. Universal accessibility of 'accessible' fitness and recreational facilities for persons with mobility disabilities. Adapt Phys Activ $Q$ 2011; 28: 1-15.

39 Dolbow DR, Figoni SF. Accommodation of wheelchair-reliant individuals by community fitness facilities. Spinal Cord 2015; 53: 515-519.
40 Carver CS, Scheier MF, Weintraub JK. Assessing coping strategies: a theoretically based approach. J Pers Soc Psychol 1989; 56: 267-283.

41 Livneh H, Martz E. Coping strategies and resources as predictors of psychosocial adapataion among people with spinal cord injury. Rehabil Psychol 2014; 59: 329-339.

42 Tawashy AE, Eng JJ, Lin KH, Tang PF, Hung C. Physical activity is related to lower levels of pain, fatigue and depression in individuals with spinal-cord injury: a correlational study. Spinal Cord 2009; 47: 301-306.

43 van den Berg-Emons RJ, L'Ortye AA, Buffart LM, Nieuwenhuijsen C Nooijen CF, Bergen MP et al. Validation of the Physical Activity Scale for individuals with physical disabilities. Arch Phys Med Rehabil 2011; 92: 923-928. 